\title{
Research Progress of the Maternal-Fetal Interface Immune Microenvironment Regulated by Traditional Chinese Medicine in the Treatment of Recurrent Spontaneous Abortion
}

\author{
Jingyi Wang1, Nan $\mathbf{L i}^{2 *}$ \\ ${ }^{1}$ Shaanxi University of Chinese Medicine, Xianyang 712000, Shaanxi Province, China \\ 2The Affiliated Hospital of Shaanxi University of Chinese Medicine, Xianyang 712000, Shaanxi Province, China \\ *Corresponding author: Nan Li, linan_80_311@163.com
}

\begin{abstract}
This article is a summary of the research progress of the maternal-fetal interface immune microenvironment regulated by traditional Chinese medicine in the treatment of recurrent spontaneous abortion. The imbalance of the immune microenvironment at the maternal-fetal interface is closely related to the occurrence of recurrent spontaneous abortion. Traditional Chinese medicine can maintain the homeostasis of the immune microenvironment at the maternal-fetal interface by regulating the function of immune cells and the expression of related cytokines.
\end{abstract}

Keywords: Maternal-fetal interface; Immune microenvironment; Traditional Chinese medicine; Research progress

Publication date: November 2021; Online publication: November 30, 2021

\section{Introduction}

Pregnancy loss within 28 weeks of three or more pregnancies is defined as recurrent spontaneous abortion (RSA) ${ }^{[1]}$. It is a disease which endangers women's reproductive health. The causes of RSA include chromosomal abnormalities, anatomical abnormalities, endocrine and metabolic disorders, semen abnormalities, etc. Even with a clear classification, the cause of approximately 50\% of RSA is unexplained (unexplained recurrent spontaneous abortion, URSA); however, $80 \%$ of URSA are considered to be closely related to immune factors ${ }^{[2]}$. Immunotherapy and cytokine regulation are the main treatment methods, but their efficacies require further confirmation. Recurrent spontaneous abortion is under the category of "habitual abortion" in traditional Chinese medicine. In recent years, traditional Chinese medicine has made gratifying progress in regulating the immune microenvironment at the maternal fetal interface. Therefore, looking back on the research results of traditional Chinese medicine and analyzing the current situation as well as shortcomings are of great significance for traditional Chinese medicine to further play a role in the field of reproductive health.

\section{Formation and function of maternal-fetal interface}

Normal pregnancy maintenance is a precise process. The maternal-fetal interface maintains the balance of immune tolerance between the mother and fetus through a series of active immune regulation ${ }^{[3]}$. The cells involved can be divided into two categories: extravillous trophoblast (EVT) from the embryo and decidual cells from the mother. EVT cells are highly invasive; after migrating and invading into the maternal decidual stroma, decidual immune cells effectively recognize them and form an immune microenvironment 
conducive to embryo implantation and development ${ }^{[4]}$.

\section{Maternal-fetal interface immune imbalance and RSA}

At the beginning of pregnancy, about $30 \%$ to $40 \%$ of decidual interstitial cells comprise of decidual lymphocytes, mainly decidual natural killer cells (dNK), dendritic cells (DC), and $\mathrm{CD}_{4}{ }^{+} \mathrm{T}$ lymphocytes. These cell groups are very important for fetal immune maintenance and the structural remodeling of the uterine spiral arteries. They are the basis of maternal-fetal interface immune tolerance.

Decidual natural killer ( $\mathrm{dNK}$ ) cells account for about $70 \%$ of decidual immune cells ${ }^{[5]}$. Unlike peripheral blood NK cells (PNK), dNK cells have relatively low cytotoxicity. Studies have shown that dNK cells produce interleukin-8 (IL-8) and interferon-gamma-inducible protein 10 (IP-10) to regulate the invasion of trophoblast. Meanwhile, dNK cells are effective secretors of a series of angiogenesis factors, which can induce decidual angiogenesis and complete spiral artery recasting ${ }^{[6]}$. Decidual macrophages account for about 20 to $30 \%$ of decidual immune cells. Decidual macrophages are a specialized cell type that performs the function of antigen presentation. In current studies, decidual macrophages are divided into M1 and M2. M2 is mainly expressed at the maternal-fetal interface, playing the role of an antiinflammatory, clearing apoptotic trophoblast, and inducing immune tolerance at the maternal-fetal interface [7] $\mathrm{CD}_{4}{ }^{+} \mathrm{T}$ lymphocytes account for about $10 \%$ to $20 \%$ of decidual immune cells. $\mathrm{CD}_{4}{ }^{+} \mathrm{T}$ cells, including helper T cells (Th) and regulatory T cells (Treg), play an important role in pregnancy maintenance. Th cells can be divided into Th1, Th2, and Th17 cells. Th2 and Treg cells play an immunosuppressive role, while Th1 and Th17 cells play a role in activating immunity ${ }^{[4]}$.

\section{Effect of traditional Chinese medicine on the immune microenvironment at the maternal-fetal interface}

In recent years, clinical and animal experimental studies have shown that traditional Chinese medicine plays an important role in regulating the immune balance at the maternal-fetal interface to prevent recurrent spontaneous abortion. The immune regulation of traditional Chinese medicine at the maternal-fetal interface is mainly reflected in the regulation of decidual NK cell function as well as the pathological imbalance of TH1/TH2 and Treg/Th17 cells.

\subsection{Regulation of decidual NK cell function and differentiation}

The regulation of decidual NK cells by traditional Chinese medicine mainly focuses on the regulation of the recruitment level and their cytokine secretion function. In a study ${ }^{[8]}$, the patients were divided into a healthy early pregnancy group, a kidney deficiency URSA pregnancy group, and a kidney deficiency URSA pregnancy loss group. Patients with kidney deficiency syndrome in the URSA group were intervened with traditional Chinese medicine. Through analyzing and comparing blood and decidua samples, it was confirmed that the inhibitory receptor of NK cells in the traditional Chinese medicine intervention group increased, and the expression of activated receptor was lower compared to that before treatment, thus proving that traditional Chinese medicine can play a role in mediating immune tolerance at the maternalfetal interface.

\subsection{Regulation of the pathological imbalance of TH1/TH2}

Jing Sun and other researchers used "Taishan Panshi Powder" in treating mice with recurrent spontaneous abortion ${ }^{[9]}$. The results showed that the traditional Chinese medicine effectively increased the level of IL10 and the expression of SOCS3 protein in the decidua of RSA mice, while the IFN- $\gamma$ level and the 
expression of SOCS1 protein at the maternal-fetal interface reduced. It has been suggested that traditional Chinese medicine can promote the shift of maternal-fetal interface to TH2 immunity and has a positive effect on the maintenance of pregnancy.

\subsection{Regulation of the pathological imbalance of Treg/Th17}

In another study, Xixi Zuo intervened RSA model mice with traditional Chinese medicine for tonifying the kidney and strengthening the spleen ${ }^{[10]}$. The results showed that the expression of cytokines IL-10 and TGF- $\beta$ increased, while IL-17A and IL-6 decreased in the TCM intervention group, indicating that the kidney-tonifying and spleen-strengthening traditional Chinese medicine can mediate the balance between Treg cells and Th17 cells by affecting their differentiation direction, thus promoting maternal-fetal tolerance.

\section{Problems and prospects}

Recurrent spontaneous abortion is under the category of "habitual abortion" in traditional Chinese medicine. The treatment advantages of traditional Chinese medicine have been widely reflected in the clinical treatment of RSA, but there are still many problems and deficiencies.

(1) RSA is often the result of the interaction of multiple causes. At present, research mostly focuses on the function of immune cells in a fixed stage. It would be beneficial to analyze the intervention of traditional Chinese medicine at different stages of pregnancy in the future.

(2) At present, the research sample size is small, and the results are inadequate to fully explain the immune mechanism. It is necessary to move in the direction of a larger sample and multicenter research.

(3) At present, most of the research is on the influence of traditional Chinese medicine on a single immune regulatory factor. The mechanism of interaction between those single factors should be studied in the future.

In conclusion, studies on the intervention of traditional Chinese medicine on the immune microenvironment at the maternal-fetal interface require multi-level and multi-channel research, so as to provide clearer medical evidence to further guide clinical practice.

\section{Funding}

This research was supported by the Discipline Innovation Team Construction Project of Shaanxi University of Chinese Medicine - Infertility Innovation Team of Shaanxi University of Chinese Medicine (Number: 2019-QN03).

\section{Disclosure statement}

The authors declare that there is no conflict of interest.

\section{References}

[1] Zhang J, 2016, Expert Consensus on the Diagnosis and Treatment of Recurrent Abortion. Chinese Journal of Obstetrics and Gynecology, 51(01): 3-9.

[2] Saravelos SH, Regan L, 2014, Unexplained Recurrent Pregnancy Loss. Obstet Gynecol Clin North Am, 41(1): 157-166. 
[3] LiX, Zhou J, Fang M, et al., 2020, Pregnancy Immune Tolerance at the Maternal-Fetal Interface. International Reviews of Immunology, (2): 1-17.

[4] Turco MY, Moffett A, 2019, Development of the Human Placenta. Development, 146(22): dev163428.

[5] Bulmer JN, Williams PJ, Lash GE, 2010, Immune Cells in the Placental Bed. Int J Dev Biol, 54(2-3): 281-294.

[6] Liu S, Diao L, Huang C, et al., 2017, The Role of Decidual Immune Cells on Human Pregnancy. J Reprod Immunol, 124: 44-53.

[7] Kieffer TEC, Laskewitz A, Scherjon SA, et al., 2019, Memory T Cells in Pregnancy. Front Immunol, 2(10): 625 .

[8] Weiping C, 2018, Study on the Effect and Mechanism of Kidney-Supplementing ThoroughfareSecuring Method on Regulating NK Cell Function and Subgroup Differentiation in the Treatment of URSA with Kidney Deficiency. Shandong University of Traditional Chinese Medicine.

[9] Sun J, Yang Z, Guo T, 2017, Intervention Effect of Taishan Panshi Powder on Immune Cell Function of Maternal-Fetal Interface in Mice with Repeated Spontaneous Abortion. Chinese Archives of Traditional Chinese Medicine, 035(010): 2689-2692.

[10] Zuo X, 2019, Analysis of Medication Rules in the Treatment of Recurrent Abortion with Traditional Chinese Medicine and Study on the Influence of Treg/Th17 Balance Mechanism. Zhejiang Chinese Medical University. 